\title{
Stabilization of a Fabry-Perot interferometer using a suspension-point interferometer
}

\author{
Y. Aso, M. Ando, K. Kawabe, S. Otsuka, K. Tsubono \\ Department of Physics, University of Tokyo, 7-3-1, Hongo, Bunkyo-ku, Tokyo, \\ Japan.
}

\begin{abstract}
A suspension-point interferometer (SPI) is an auxiliary interferometer for active vibration isolation, implemented at the suspension points of the mirrors of an interferometric gravitational wave detector. We constructed a prototype Fabry-Perot interferometer equipped with an SPI and observed vibration isolation in both the spectrum and transfer function. The noise spectrum of the main interferometer was reduced by $40 \mathrm{~dB}$ below $1 \mathrm{~Hz}$. Transfer function measurements showed that the SPI also produced good vibration suppression above $1 \mathrm{~Hz}$. These results indicate that SPI can improve both the sensitivity and the stability of the interferometer.
\end{abstract}

Key words: gravitational wave, active vibration isolation, suspension point interferometer PACS: 04.80.Nn, 95.55.Ym

\section{Introduction}

Several large-scale laser interferometric gravitational wave detectors, such as TAMA [1], LIGO [2], GEO [3] and VIRGO [4], are under construction or have started scientific observations [5][6][7][8]. The main targets of these detectors are violent astronomical events, such as the coalescence of neutron-star binaries [9]. However, such kinds of events that produce strong gravitational radiation are extremely rare [10]. To increase the event rate, we need to improve the interferometers to be sensitive to events from a larger volume of space. At the same time, the small event rate requires us to operate the interferometers stably over the long term in a good condition.

Email address: aso@granite.phys.s.u-tokyo.ac.jp (Y. Aso,). 


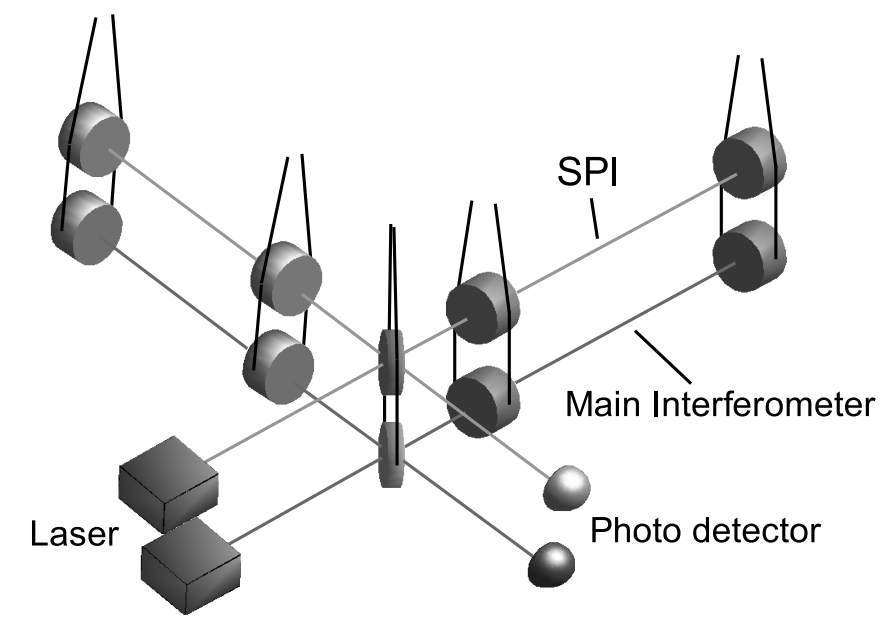

Fig. 1. Fabry-Perot-Michelson interferometer with SPI. The lower interferometer (main interferometer) is used to detect gravitational waves and the upper one is mainly used for active vibration isolation.

A suspension-point interferometer (SPI) is an active vibration isolation scheme that improves both the sensitivity and the stability of an interferometer[11]. Fig. 1 shows the basic configuration of a Fabry-Perot-Michelson interferometer equipped with an SPI. The lower interferometer is called the main interferometer and is used to detect gravitational waves. The upper interferometer (SPI) is mainly used for active vibration attenuation.

SPI was originally proposed by Drever more than 20 years ago as an advanced vibration isolation scheme [12]. He demonstrated the stabilization of an asymmetric Michelson interferometer with corner cube mirrors using an SPI [13]. However, further experimental study on the feasibility and the practical limits of SPI, especially using Fabry-Perot interferometers, was needed for applications of this technique to large-scale interferometers. For this purpose, we constructed a prototype Fabry-Perot interferometer equipped with an SPI, strongly motivated by its possible applications to LCGT [14], the cryogenic interferometer project in Japan.

The purpose of this paper is to present results from the prototype experiment to demonstrate the basic features of SPI. In the next section, we explain the working principle and the performance limits of SPI. We then describe the experimental setup of the prototype interferometer, and in the following section discuss the results from the experiment. 


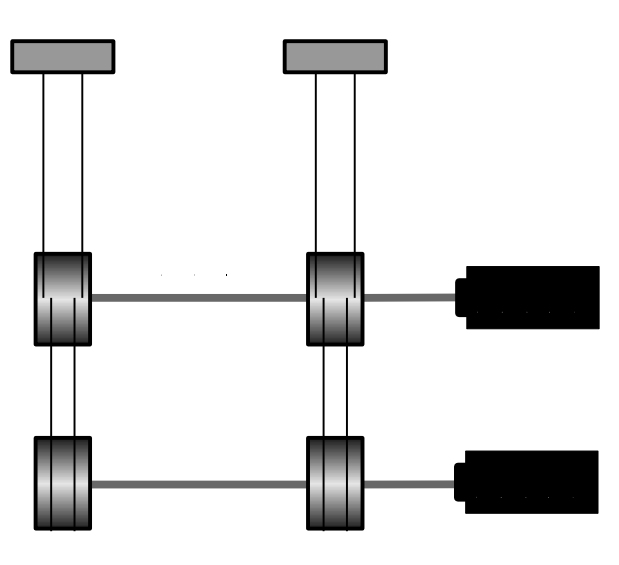

$-(\mathrm{a})-$

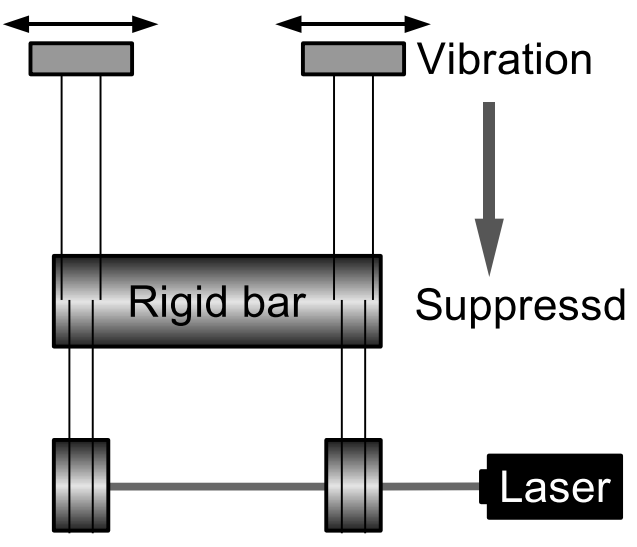

$-(\mathrm{b})-$

Fig. 2. Working principle of SPI. When the upper interferometer (SPI) is locked, that stage can be regarded as being a rigid bar. This rigid bar resists differential external disturbances.

\section{Suspension-Point Interferometer}

In the following section, the working principle of SPI is explained first. We then discuss the advantages of SPI: the reduction of the residual motion, and the low noise nature of SPI. The applications of SPI to cryogenic interferometers and lock acquisition of interferometers are also discussed. In section 2.2, the theoretical performance limits of SPI are considered.

\subsection{Working principle}

To explain the working principle of SPI, let us first consider one arm of a Fabry-Perot-Michelson interferometer (Fig. 2(a)). When we apply forces to lock the upper interferometer (SPI) to the injected laser, the fluctuation of the distance between the two mirrors of the SPI is suppressed by its control servo. Therefore, those mirrors form a virtual rigid bar, as shown in Fig. 2(b). This virtual rigid bar resists external disturbances to change its length. Consequently, the SPI reduces the transmission of seismic noise to the main interferometer. In the full configuration, as shown in Fig. 1, each arm cavity is stabilized by an SPI. Consequently, the combination of those stabilized cavities forms a stabilized Fabry-Perot-Michelson interferometer.

In addition to the reduction of seismic noise, we can also improve the stability of the interferometer using SPI. When we lock the SPI, the mirrors of the main interferometer behave as single pendulums suspended from a rigid bar (Fig. 2(b)). From now on we call these single pendulums the "main pendu- 
lums". This simplification of the suspension system introduced by SPI makes it possible to suppress most of the resonances of the complex multi-stage suspension system. As a result, we can reduce the residual motion of the mirrors, and thus improve the stability of the interferometer. Also, the smaller residual motion allows us to use weaker coupling actuators for control of the mirrors. This reduces the actuator noise, one of the most serious technical noise sources in advanced interferometers.

Generally speaking, the performance of an active vibration isolation system is limited by the noise of the sensor. However, in the case of SPI, the sensor is an interferometer, which potentially has the same sensitivity as the main interferometer, itself. Therefore, the performance of SPI is not limited by the sensor noise. We consider the performance-limiting factors of SPI later.

The low-noise feature of SPI is particularly useful for cryogenic interferometers, such as LCGT. To realize a cryogenic interferometer, we have to attach heat-link wires as close to the mirrors as possible in order to extract heat efficiently. However, the heat-link wires also introduce additional seismic vibration to the suspension stage to which the wires are attached [15]. To suppress this vibration, we need a very low-noise active vibration attenuation scheme, because any noise produced by the active system is transmitted to the main mirrors with only a few stages of isolation. The low-noise nature of SPI is suitable for this purpose.

SPI is also useful as a tool to help acquire the locked condition of an interferometer, especially for the RSE (Resonant Sideband Extraction) interferometer [16], which is an advanced interferometer configuration planned to be used in next generation interferometers. Although it is generally difficult to lock highfinesse cavities used in RSE interferometers, reduction of the residual motion of the mirrors introduced by SPI will make it easy to acquire the locked condition.

\subsection{Performance limits}

The vibration isolation ratio of SPI is mainly determined by three factors: the control gain, common mode rejection ratio (CMRR), and coupling from other degrees of freedom. Using the gain $G$ of the length control servo, the vibration attenuation factor by an SPI can be written as $1 /(1+G)$. Usually $G$ can be set sufficiently large $\left(\sim 10^{6}\right.$ in our experiment) at frequencies where the seismic vibration is significant, typically below $10 \mathrm{~Hz}$. Therefore, in practice the other two factors are the dominant limitations to the performance of SPI.

First, we consider CMRR. Since Fabry-Perot interferometers are only sensitive

to the differential motion of their mirrors, the common motion of the mirrors 
is not suppressed by SPI and is transmitted to the main interferometer with the only attenuation of a single pendulum. In an ideal case, the common motion does not produce any noise in the main interferometer, which is also insensitive to common motion. However, in the real case, a fraction of the common motion of the SPI mirrors is converted into the differential motion of the main mirrors due to asymmetries in the main pendulums.

The CMRR of SPI is defined as the ratio of this converted differential motion to the common motion; CMRR represents the amount of vibration isolation produced by an SPI. Using the transfer functions from the suspension point to the masses of the main pendulums, $H_{1}$ and $H_{2}$, CMRR can be written as

$$
\operatorname{CMRR}=2\left|\frac{H_{1}(\omega)-H_{2}(\omega)}{H_{1}(\omega)+H_{2}(\omega)}\right| .
$$

In the point-mass approximation, $H_{1}$ and $H_{2}$ are explicitly written as

$$
\begin{aligned}
& H_{1}(\omega)=\frac{1}{1+i \frac{l_{1} \Gamma_{1}}{m_{1} g} \omega-\frac{l_{1}}{g} \omega^{2}}, \\
& H_{2}(\omega)=\frac{1}{1+i \frac{l_{2} \Gamma_{2}}{m_{2} g} \omega-\frac{l_{2}}{g} \omega^{2}},
\end{aligned}
$$

where $l_{1}$ and $l_{2}$ are the lengths of the main pendulums, $m_{1}$ and $m_{2}$ are the masses of the mirrors, $\Gamma_{1}$ and $\Gamma_{2}$ are the damping coefficients of the pendulums, and $g$ is the gravitational constant. Combining Eqs. (1),(2) and (3), we obtain the following expression:

\section{$\mathrm{CMRR} \simeq$}

$$
\left|H_{1}(\omega)\right| \sqrt{\left(\frac{\Delta l}{g}\right)^{2} \omega^{4}+\left(\frac{l \Gamma}{m g}\right)^{2}\left(\frac{\Delta l}{l}+\frac{\Delta \Gamma}{\Gamma}-\frac{\Delta m}{m}\right)^{2} \omega^{2}}
$$

where, $l, m$, and $\Gamma$ denote the mean values of the length, the mass, and the damping coefficient of the two pendulums, respectively. In this approximation, only the first and second order terms of $\Delta l \equiv l_{1}-l_{2}, \Delta m \equiv m_{1}-m_{2}$, and $\Delta \Gamma \equiv \Gamma_{1}-\Gamma_{2}$ were taken into account.

At frequencies higher than the resonant frequency of the main pendulums, CMRR approaches $\Delta l / l$ (Fig. 3). Therefore, the vibration attenuation factor by SPI is limited by $\Delta l / l$ in this frequency region. For example, if the asymmetry of the wire length is $1 \%$, the limit vibration isolation ratio of the SPI is $40 \mathrm{~dB}$. Below the resonant frequency of the main pendulums, the CMRR decreases as the frequency goes down.

Coupling from other degrees of freedom sets another limit to the performance of SPI. SPI is only sensitive to the length change of the interferometer, which is 


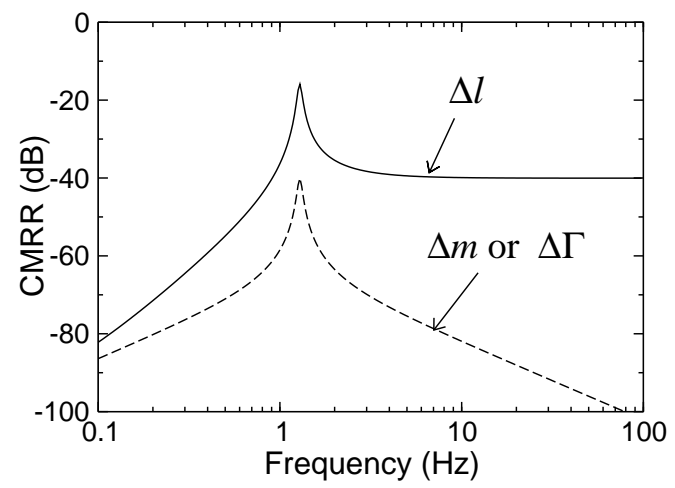

Fig. 3. The CMRR of an SPI with $1 \%$ asymmetry. The solid line shows CMRR with $\Delta l=1 \%$. The CMRR approaches to $\Delta l / l$ at frequencies above $1.3 \mathrm{~Hz}$, which is the resonant frequency of the pendulums. The dashed line shows CMRR with $\Delta m=1 \%$, or $\Delta \Gamma=1 \%$. From the symmetry of Eq. (4), the dependence of CMRR to $\Delta m$ and $\Delta \Gamma$ is identical.

caused by the horizontal motion of the mirrors along the direction of the laser beam. Therefore, the motions of mirrors transverse to the beam direction, such as vertical vibration, are not suppressed by SPI. Just like the case of CMRR, a fraction of those motions is converted to the motion along the beam direction by asymmetries in the suspension system and by the convergence of the local vertical lines towards the center of the earth.

In the case of LCGT, the amount of vertical-to-horizontal coupling caused by the curvature of the earth is $0.2 \%$ [17]. A fraction of rotational motions is also coupled to the length fluctuation of the interferometer, through a beam miscentering and vertical offsets of the wire release points from the mirror's center of gravity. Considering that the amount of those miscentering and offsets is less than $1 \mathrm{~mm}$ in TAMA [18], the coupling coefficient from rotational motions to the beam direction is smaller than $10^{-3} \mathrm{~m} / \mathrm{rad}$. However in real suspension systems, it is not simple to calculate the total amount of coupling from other degrees of freedom, because it also depends on various manufacturing imperfections. Typical measured coupling coefficients are on the order of $1 \%[17]$.

\section{Experimental setup}

To test the basic features of SPI, we constructed a prototype interferometer equipped with an SPI. As a test interferometer, we used a Fabry-Perot interferometer instead of a full configuration Fabry-Perot-Michelson interferometer, because it is sufficient to test the working principle and the basic performance of SPI. 


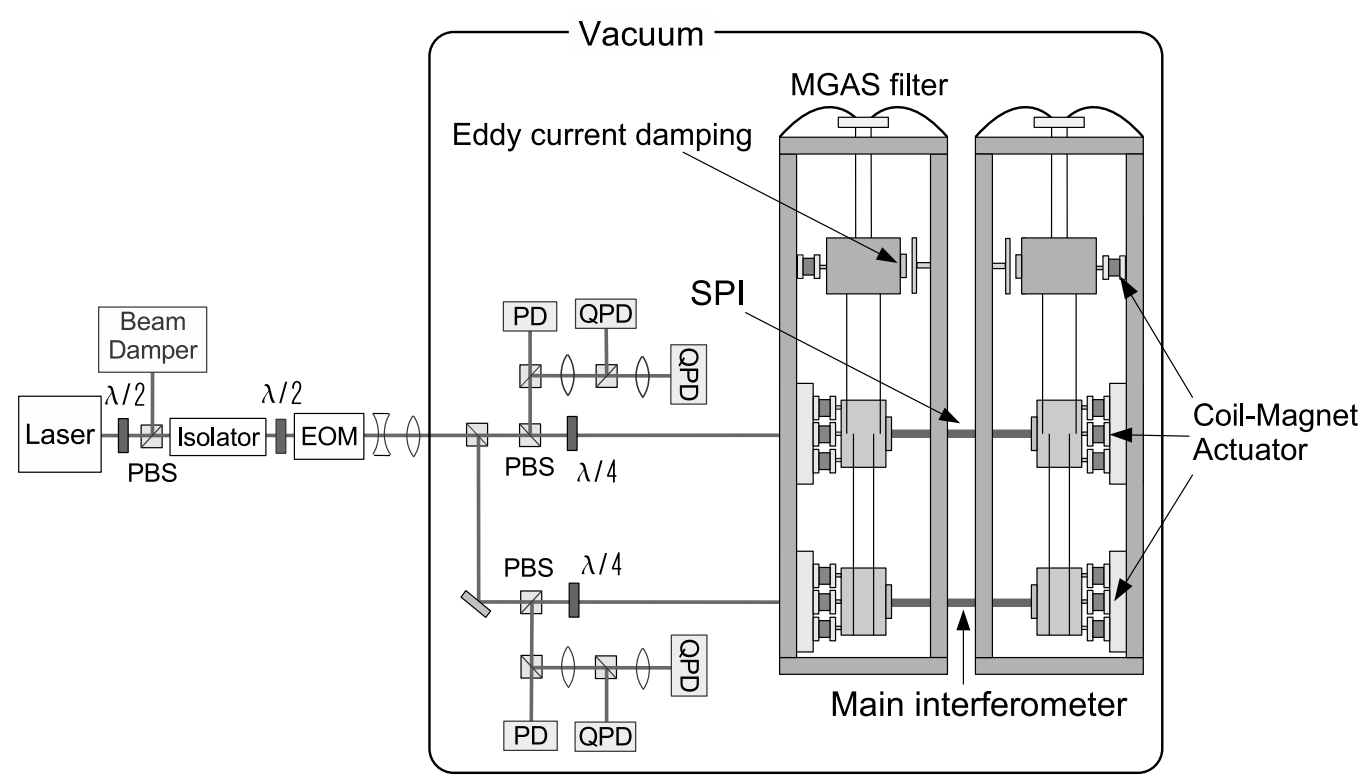

Fig. 4. Experimental setup. The main interferometer is a Fabry-Perot interferometer suspended from another Fabry-Perot interferometer (SPI). Light from a laser is divided by a beam splitter and injected into each interferometer. The reflected laser beams are detected by several photo-detectors to obtain the length and alignment signals.

Fig. 4 is a schematic drawing of the experimental apparatus. The main interferometer is suspended from an SPI. Each interferometer is a $15 \mathrm{~cm}$ long Fabry-Perot interferometer with a finesse of 200. Our objective is to see a reduction of the length fluctuation of the main interferometer by locking the SPI.

The suspension system of the interferometers is a triple pendulum with a total length of $50 \mathrm{~cm}$. The final stage and the second stage of the suspension form the main and the suspension point Fabry-Perot interferometers, respectively. The masses of the first stage are called damping masses; eddy current damping [19] is applied to this stage to reduce the residual motion of the mirrors. We used MGAS filters [20], a low-frequency vertical spring, for vertical vibration isolation. Coil-magnet actuators are attached to each mirror in order to control their position and orientation. We also attached coil-magnet actuators to the damping masses to excite them and measure the transfer function from the damping stage to the main interferometer.

We used a $500 \mathrm{~mW}$ NPRO Nd:YAG laser with integrated temperature and intensity stabilization systems. The power of the laser was attenuated to 80 $\mathrm{mW}$ to avoid saturation of the photo detectors. The light emitted by the laser is phase-modulated at $15 \mathrm{MHz}$ by an EOM after a Faraday isolator. After passing through mode matching lenses, the light is injected into a vacuum chamber. 


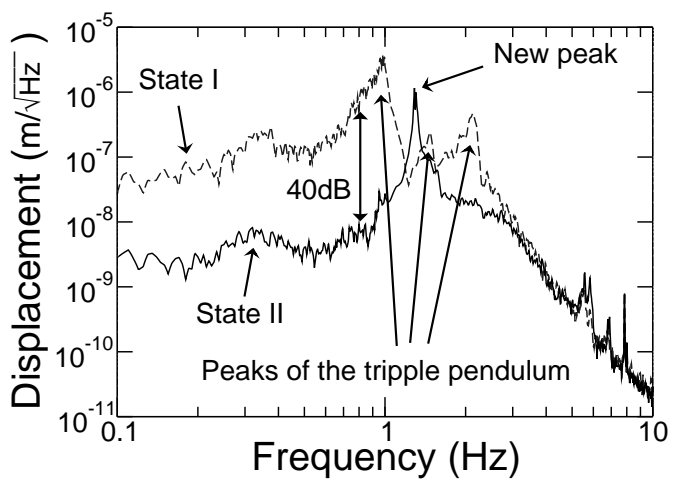

Fig. 5. Equivalent displacement noise spectra of the main interferometer. The dashed and solid lines are the spectrum in state I and state II, respectively. Maximum $40 \mathrm{~dB}$ of noise reduction is observed in state II. The number of resonant peaks is reduced from 3 in state I to 1 in state II.

Inside the chamber, the beam is divided into two beams by a beam splitter, and fed to the main and suspension-point cavities. The reflected light from each of the cavities is divided by beam splitters and then detected by single-aperture photo detectors and quadrant photo detectors. The error signals of the length of the each cavity are obtained by the Pound-Drever-Hall (PDH) method [21] using signals from the single-aperture photo detectors. The quadrant photo detectors are placed at different Guoy phases of the reflected beams to provide the alignment information of the cavities with the wave-front sensing (WFS) technique [22][23]. Two pairs of lenses are used to adjust the Guoy phases.

The lengths of the interferometers are locked to the laser by feeding the PDH signals back to the coil-magnet actuators on the mirrors through appropriate servo filters. The unity gain frequency (UGF) of the length control servo loop is about $1 \mathrm{kHz}$ in both interferometers, and the phase margin at UGF is about 45 degrees. The open-loop gain at DC is more than $120 \mathrm{~dB}$. The interferometers are remarkably stable and continuously locked for more than 10 hours. Actually, they have never lost the locked state unless the servo is intentionally turned off. The alignment of the mirrors is also controlled by feeding the WFS signals back to the coil-magnet actuators. The bandwidth of the WFS servo is about $30 \mathrm{~Hz}$. Note that the SPI works as an attenuator for the rotational as well as longitudinal motion of the mirrors, because of this WFS servo.

\section{Results}

In this section we show the experimental results and compare the two operation modes of the interferometers. The first operation mode is called state I, in which we only operate the main interferometer, and the servo loop of the SPI is turned off. The second operation mode is called state II, in which we 
lock both the SPI and the main interferometer. In state II, the SPI works as a vibration isolation device. We have made three types of measurements: i) noise spectrum measurements of the main interferometer, ii) transfer function measurements of the cavity length, iii) transfer function measurements of the angular motion of the mirrors.

Fig. 5 shows the measured length fluctuation spectra of the main interferometer between $0.1 \mathrm{~Hz}$ and $10 \mathrm{~Hz}$. The dashed curve is the spectrum measured in state I. The solid curve is the spectrum measured in state II. By comparing these two curves, one can see that the noise of the main interferometer is suppressed by the SPI below $3 \mathrm{~Hz}$. The maximum suppression ratio is $40 \mathrm{~dB}$, around $1 \mathrm{~Hz}$. The root mean square (RMS) of the mirror motion integrated from $0.1 \mathrm{~Hz}$ to $10 \mathrm{~Hz}$ is $1 \times 10^{-6} \mathrm{~m}$ in state $\mathrm{I}$, and $2 \times 10^{-7} \mathrm{~m}$ in the state II. The RMS motion of state II is 5 times smaller than that of state I.

The three peaks in state I spectrum, which correspond to the resonances of the triple pendulum suspension, are well suppressed by the SPI and disappear in state II. Instead, a sharp peak appears at $1 \mathrm{~Hz}$ in state II, which is the resonance of the main pendulum. This results can be understood by the rigidbar picture of SPI, explained in section 2.1. Although the new peak in state II is very sharp, it does not make the interferometer unstable. In fact, the peak does not appear in the feedback signal to the actuators, because the actuation efficiency in state II has an equally sharp peak at exactly the same frequency, and these peaks cancel out each other. In other words, the new peak is well suppressed by the feedback system of the main interferometer.

Fig. 6 shows transfer functions of the longitudinal motion from one of the damping masses to the main interferometer. The dashed curves are theoretical curves calculated by a simple point-mass model of the suspension system. To calculate the theoretical curve for state II, we adjusted the amount of the asymmetries in the model suspension to match the measured height of the peak at $1.2 \mathrm{~Hz}$.

We observed the improvement of the vibration isolation ratio by the SPI at frequencies below $5 \mathrm{~Hz}$. In the frequency region below the resonant frequency of the main pendulums, i.e. below $1 \mathrm{~Hz}$, the isolation ratio increases as the frequency decreases. The measured behavior qualitatively agrees with the CMRR calculation shown in Fig. 3. However, there is some discrepancy between the measurement and the theoretical curve. The most-likely reason of this disagreement is some kind of cross talk in the measurement system. However, we do not currently have any definitive explanation for this discrepancy, and further investigation is needed.

At frequencies above $5 \mathrm{~Hz}$, we could not measure the transfer function properly due to asymmetry in the actuators on the damping mass, that excites 


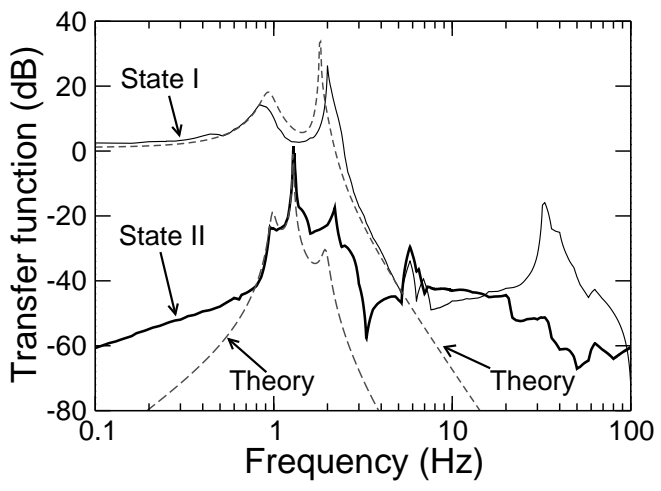

Fig. 6. Transfer functions from the displacement of a damping mass to the main interferometer. The damping mass was excited by means of coil magnet actuators and the transferred displacement was measured by the main interferometer. At 0.1 $\mathrm{Hz}$, the vibration isolation ratio provided by SPI is $60 \mathrm{~dB}$.

rotational motion of the damping mass. The ratio of the transfered rotational motion to the horizontal motion at the main interferometer becomes larger at higher frequencies, because the resonant frequencies of the rotational modes of the masses are higher than that of the pendulum. The peaks at $6 \mathrm{~Hz}$ and $35 \mathrm{~Hz}$ correspond to the yaw and pitch $^{1}$ resonances of the main mirror. Since the beam spots on the mirrors are not perfectly centered, the rotational motion couples to the length change of the interferometers and contaminates the transfer function measurements. With more careful design and adjustment of the actuator balance, we should be able to see a better improvement in the vibration isolation ratio.

Fig. 7 shows the transfer functions of the angular motion of the mirrors. The yaw motion of one of the damping masses was excited by the coil magnet actuators and the transfered yaw motion was measured by the WFS of the main interferometer. The theoretical curves are calculated using a simple rigidbody model of the suspension system.

The measured transfer functions are in good agreement with the theory at frequencies below $8 \mathrm{~Hz}$. Comparing the transfer functions of state I (the WFS servo of the SPI is off) and state II (the WFS servo of the SPI is on), one can see that the maximum suppression ratio is $30 \mathrm{~dB}$, which corresponds to the DC gain of the WFS servo of the SPI. Above $8 \mathrm{~Hz}$ the measurement is probably limited by cross talk in the electric circuits.

1 "Yaw" means rotation about the vertical axis. "Pitch" means rotation about the horizontal axis which is perpendicular to the beam direction. 


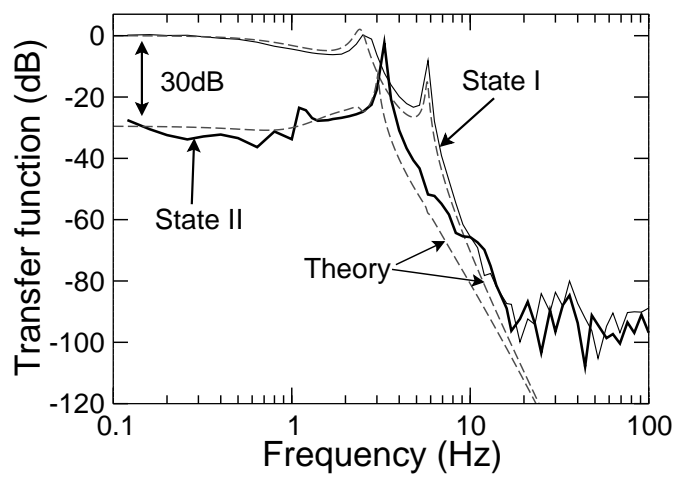

Fig. 7. Transfer functions of the yaw motion from a damping mass to a mirror of the main interferometer. The damping mass is excited by coil magnet actuators and the yaw motion of the main mirror of the same suspension chain is measured by the WFS signal of the main interferometer. The maximum suppression ratio is $30 \mathrm{~dB}$; this is the gain of the alignment servo of the SPI.

\section{Discussion}

From Fig. 6, we can see that the vibration isolation ratio of the SPI is about $60 \mathrm{~dB}$ at $0.1 \mathrm{~Hz}$. However, in the noise power spectrum shown in Fig. 5, the difference between state I and II is only slightly above $20 \mathrm{~dB}$ at $0.1 \mathrm{~Hz}$. Besides, at frequencies above $3 \mathrm{~Hz}$ we can see no difference in the noise spectrum, though the servo gain of the SPI is still high at those frequencies. We suppose that the best explanation for these discrepancies is vertical vibration, because other possible reasons, such as electric noise and laser frequency noise, are excluded by the reasoning given below.

Firstly, in the whole frequency region shown in Fig. 5, the measured electric noise was well below that of the main interferometer. Secondly, the effective length change induced by the frequency noise is coherent in both interferometers. Below the resonant frequency of the main pendulum, where the length of the main interferometer follows that of the SPI, this effective length change of the main interferometer is canceled by the SPI. Therefore, the frequency noise should be well suppressed at those frequencies. Above the resonance of the main pendulum, the frequency noise is not canceled by the SPI, because the coupling between the mirror motion of the two interferometers becomes weaker. Instead, the noise of the two interferometers will be correlated, if the dominant noise source is the frequency noise. However, we observed no correlation between the noise of the two interferometers at those frequencies. Hence, the dominant noise source in this frequency range can not be the laser frequency noise. To confirm this, we subtracted the error signals from both interferometers. If the two signals are correlated, they should cancel out at least partially. To determine the ratio of the subtraction, we put a sinusoidal signal on the PZT frequency tuning of the laser to introduce a peak in the error 
signals, and adjusted the ratio so that the peak would disappear after subtraction. With this operation, no signal cancellation was observed. Therefore, we can exclude both electric and frequency noises.

To obtain better performance of the SPI, we need better vertical vibration isolation. We have developed an active vibration suppression device for vertical motion using a vertical suspension point interferometer[24].

Since SPI can have basically the same sensitivity as the main interferometer, we can also use the SPI for the detection of gravitational waves at high frequencies where seismic noise is not significant, leaving the detection of lower frequency gravitational waves to the main interferometer. Study on this idea is also on going [25].

\section{Summary}

A suspension point interferometer is useful for stabilization and a sensitivity improvement of large scale interferometers. Especially in cryogenic interferometers, SPI can be used for the attenuation of the vibration introduced by heat link-wires.

We have constructed and stably operated a Fabry-Perot interferometer equipped with an SPI. Using the SPI, the length fluctuation spectrum of the main interferometer has been reduced by $40 \mathrm{~dB}$ at maximum, and the RMS of the length fluctuation has been suppressed by a factor of 5 . The resonant peaks of the triple pendulum suspension have also been suppressed by the SPI. We believe that we can obtain a better seismic attenuation performance with better vertical vibration isolation.

\section{Acknowledgement}

We are grateful to Riccardo DeSalvo and Akiteru Takamori for providing us with their MGAS filters. A part of this research is supported by a Grant-in-Aid for JSPS Fellows and a Grant-in-Aid for Scientific Research on Priority Areas (415) of the Ministry of Education, Culture, Sports, Science and Technology. 


\section{References}

[1] K. Tsubono, 300-m Laser Interferometer Gravitational Wave Detector (TAMA300) in Japan, in: E. Coccia, G. Pizzella, F. Ronga (Eds.), Proc. First E. Amaldi Conference on Gravitational Wave Experiments, June 1994, World Scientific, Singapore, 1995, p.112.

[2] A. Abramovici, W.E. Althouse, R.W.P. Drever, Y. Gürsel, S. Kawamura, F.J. Raab, D. Shoemaker, L. Sievers, R.E. Spero, K.S. Thorne, R.E. Vogt, R. Weiss, S.E. Whitcomb, M.E. Zucker, Science 256 (1992) 325

[3] K. Danzmann, H. Lück, A. Rüdiger, R. Schilling, M. Schrempel, W. Winkler, J. Hough, G.P. Newton, N.A. Robertson, H. Ward, A.M. Campbell, J.E. Logan, D.I. Robertson, K.A. Strain, J.R.J. Bennett, V. Kose, M. Kühne, B.F. Schutz, D. Nicolson, J. Shuttleworth, H. Welling, P. Aufmuth, R. Rinkleff, A. Tünnermann, B. Willke, Proposal for a $600 \mathrm{~m}$ LaserInterferometric Gravitational Wave Antenna, Report 190, Max-Planck-Institut für Quantenoptik, Garching, Germany, 1994.

[4] VIRGO collaboration, VIRGO Final Design Report, 1997.

[5] M. Ando, K. Arai, R. Takahashi, et al., Phys. Rev. Lett. 86 (2001) 3950.

[6] H. Tagoshi, T. Tanaka, N. Kanda, D. Tatsumi, S. Telada, M. Ohashi, Int. J. Mod. Phys. D 9 (2000) 319

[7] B. Abbott, R. Abbott R. Adhikari, The LIGO Scientific Collaboration, et al., Nucl. Instrum. Meth. A 517 (2004) 154.

[8] M. Hewitson, P. Aufmuth, C. Aulbert, et al., Classical Quant. Grav. 20 (2003) 581.

[9] L. Ju, D.G. Blair, C. Zhao, Rep. Prog. Phys. 63 (2000) 1317.

[10] V. Kalogera, C. Kim, D.R. Lorimer, M. Burgay, N. D’Amico, A. Possenti, R.N. Manchester, A.G. Lyne, B.C. Joshi, M.A. McLaughlin, M. Kramer, J.M. Sarkissian, F. Camilo , Astrophys. J. 601 (2004) L179.

[11] R.W.P. Drever, Outline of a proposed design for a first receiver for installation in the long-baseline facilities of Fabry-Perot type, LIGO Document T87000100-R, 1987.

[12] R.W.P. Drever, Personal communication.

[13] R.W.P. Drever, S.J. Augst, Classical Quant. Grav. 19 (2002) 2005.

[14] K. Kuroda, M. Ohashi, S. Miyoki, et al., Classical Quant. Grav. 20 (2003) 871.

[15] K. Kasahara, T. Tomaru, T. Uchiyama, T. Suzuki, K. Yamamoto, S. Miyoki, M. Ohashi, K. Kuroda, T. Shintomi, J. Cryo. Soc. Jpn. 39 (2004) 25.

[16] J. Mizuno, K.A. Strain, P.G. Nelson, J.M. Chen, R. Schilling, A. Rüdiger, W. Winkler, K. Danzmann, Phys. Lett. A 175 (1993) 273. 
[17] K. Arai, Master's thesis, University of Tokyo, 1997.

[18] A. Takamori, Master's thesis, University of Tokyo, 1998.

[19] K. Tsubono, A. Araya, K. Kawabe, S. Moriwaki, N. Mio, Rev. Sci. Instrum. 64 (1993) 2237.

[20] G. Cella, R. DeSalvo, V. Sannibale, H. Tariq, N. Viboud, A. Takamori, Nucl. Instrum. Meth. A 487 (2002) 652.

[21] R.W.P. Drever, J.L. Hall, F.V. Kowalski, J. Hough, G.M. Ford, A.J. Munley, H. Ward, Appl. Phys. B 31 (1983) 97.

[22] E. Morrison, B.J. Meers, D.I. Robertson, H. Ward, Appl. Opt. 33 (1994) 5041.

[23] E. Morrison, B.J. Meers, D.I. Robertson, H. Ward, Appl. Opt. 33 (1994) 5037.

[24] Y. Aso, A paper in preparation.

[25] Y. Aso, Talk at 2004 Aspen Winter Conference on Gravitational Waves, Aspen Center for Physics, Colorado, USA, Feb. 2004. 